\title{
Getting tense about relativity
}

\author{
James Read ${ }^{1}\left[\right.$ Emily Qureshi-Hurst $^{1}$
}

Received: 8 October 2019 / Accepted: 28 January 2020 / Published online: 4 February 2020 (c) The Author(s) 2020

\begin{abstract}
Special relativity has been understood by many as vindicating a tenseless conception of time, denying the existence of tensed facts and a fortiori objective temporal passage. The reason for this is straightforward: both passage and the obtaining of tensed facts require a universal knife-edge present moment-yet this structure is not easily reconcilable with the relativity of simultaneity. The above being said, the prospects for tense and passage are sometimes claimed to be improved on moving to cosmological solutions of general relativity. In this paper, we evaluate in detail these arguments, finding that there remain several open questions to be addressed if the introduction of tensed facts into the relativistic context is to be compelling. Moreover, we argue that, even setting aside these issues, objective tense stands and falls in relativity for exactly the same reasons that it does in classical philosophical discussions on the matter.
\end{abstract}

Keywords Tense $\cdot$ A-theory $\cdot$ Presentism $\cdot$ General relativity $\cdot$ Cosmology $\cdot$ Modal argument

\section{Introduction}

The special theory of relativity (SR) is often taken to constitute good evidence against absolute time-where 'absolute time' is defined as enshrining a universal present moment, objective temporal passage, and tensed facts. This position falls broadly under the guise of an 'A-theory' of time. The alternative 'B-theory' holds that there are no objectively tensed facts, as there is no ontological difference between what we call past, present and future to ground the truth of these facts. ${ }^{1}$

\footnotetext{
1 The positions of the 'A-theory' and 'B-theory' track, but also transcend, McTaggart's 'A-series' (that ordering of events according to whether they are past/present/future) and 'B-series' (that ordering of events according to whether they are earlier than/later than). Cf. McTaggart (1908).

$凶 \quad$ James Read

james.read@philosophy.ox.ac.uk

Emily Qureshi-Hurst

emily.qureshi-hurst@pmb.ox.ac.uk

1 Pembroke College, University of Oxford, Oxford OX1 1DW, UK
} 
There are three central A-theoretic stances which one might adopt, depending upon one's stance on the metaphysics of time. The first, 'presentism', claims that the only things that exist are the events that occur on the present's knife-edge. ${ }^{2}$ Existence entails being present, and only and all that exists are presently occurring things and events. The second is the 'growing block' view, echoing presentism's claims about the knifeedge present which is the frontier of becoming. It differs, however, in that it does not restrict existence claims to the present but allows the accumulation of real past events in a 'block' which grows as ever more moments and events come into existence and move from being present into the past. C.D. Broad, a growing block theorist, defines this view as "[accepting] the reality of the present and the past, but [holding] that the future is simply nothing at all."3 As the present becomes past, "fresh slices of existence have been added to the total history of the world[, therefore] the past is as real as the present." 4 The third central A-theoretic stance, the 'moving spotlight' view, claims that all events in time are equally real, but each is endowed with the extra, tensed properties of being past, present, or future, and these properties can change with time. 5

Whichever A-theory one adheres to, the knife-edge present moment requires the existence of absolute simultaneity-i.e., agent-independent facts about which events count as being simultaneous. This, however, immediately leads to a tension with SR, according to which (assuming for simplicity Einstein-Poincaré synchrony throughout $^{6}$ ) simultaneity itself is a frame-relative matter. Simultaneity planes depend on one's frame of reference, which means that observers in different frames of reference will not always agree on which events are simultaneous with the present-viz., occurring now. As Kurt Gödel explains,

The instance of an objective lapse of time ... means ... that reality consists of an infinity of layers of "now" which come into existence successively. But, if simultaneity is something relative ... reality cannot be split up into such layers in an objectively determined way. Each observer has his own set of "nows," and none of these various layers can claim the prerogative of representing an objective lapse of time. ${ }^{7}$

In this passage, Gödel draws out precisely the problem that the relativity of simultaneity poses for the A-theory. The A-theory depends on events possessing the objective tensed properties past, present, future, and on time being dynamic in the sense that it genuinely passes. The relativity of simultaneity stands in tension with the claim that there are objective tensed facts, because the present is defined differently for different observers. Therefore, the designation of an event as present is, at least in some sense, subjective. This is strong evidence in favour of the B-theorist's denial of tensed facts, and supports the metaphysical position that all events coexist by grounding their coexistence in the

\footnotetext{
2 Mozersky (2011, p. 122).

3 Broad (1923, p. 66).

4 Broad (1923, p. 66).

5 Skow (2015, ch. 4).

6 See e.g. Brown (2005) for discussion.

7 Gödel (1949, p. 558).
} 
four-dimensional spacetime manifold (the 'block'). The B-theorist maintains that now is not an objective property, and events do not objectively possess the property of being present. There is a natural sense in which SR supports this view. Over and above the implications of this for the existence of tensed facts, it raises serious problems for the very existence of an objective present. ${ }^{8}$

In light of these issues, A-theorists have had recourse to a range of tactics. One is to augment SR with a fact of the matter about which frame of reference is objective: this is the strategy of e.g. Lane Craig. ${ }^{9}$ We will not discuss this option further in this paper. ${ }^{10}$ A second option is to turn to the general theory of relativity (GR), and in particular to cosmological solutions thereof. In such solutions-unlike in SR-an objective foliation of the four-dimensional universe into simultaneity surfaces of constant time appears to be possible: this is known as cosmic simultaneity, and the associated temporal parameter orthogonal to such surfaces is known as cosmic time. ${ }^{11}$ If this correct, then the A-theorist would appear to be saved: certain solutions of GR (including, importantly, the solution which we take to correspond to the actual world) are equipped with sufficient structure with respect to which to ground an objective past/present/future distinction. $^{12}$

Critiques of this latter strategy have been mustered from a number of directions. First, one might argue that to focus thus on cosmological solutions of GR is, in fact, to change the theory under consideration - thereby illegitimately disengaging us from the relevant domain of discourse. ${ }^{13}$ Second, one might object that cosmological solutions of GR are mere idealisations, and are therefore not true descriptions of the actual world-in which case, one cannot appeal to the structure of such solutions in order to ground an objective past/present/future distinction in the actual world. ${ }^{14}$ Third, one might argue that to use cosmic simultaneity as a vehicle for grounding an objective past/present/future distinction is ill-conceived - for the original motivation to introduce primitive tensed properties was to recover our agential experience of the flow of time-yet cosmic time is radically disconnected from that experience. ${ }^{15}$ Finally, one might follow Gödel ${ }^{16}$ in presenting a modal argument, to the effect that whether a universe manifests primitive tensed properties cannot be a contingent affair-but

\footnotetext{
8 It often goes unnoticed in the literature that even the B-theorist will be pushed to some revision of her views in light of SR. The reason is that, while timelike-separated events will stand in objective earlier/later than relations to one another, such is not the case for spacelike-separated events. Thus, SR reveals to the B-theorist that the class of events standing in the earlier than/later than relation to one another is more impoverished than one may have hitherto anticipated.

9 See Craig (2000a, b, 2001a, b). For a critical appraisal of Lane Craig's work, see Balashov and Janssen (2003). On this issue, our sympathies lie with Balashov and Janssen — though nothing further will be made of this in the present piece.

10 Though cf. Swinburne's response in the case of general relativity, discussed in Sect. 6.2 below.

11 Swinburne (2008).

12 It has been suggested that the emergence of cosmic simultaneity in some solutions of GR is an instance of spontaneous symmetry breaking. See Arkani-Hamed et al. (2005).

13 Monton (2006). For a reply, see Wüthrich (2010).

14 Smeenk (2013).

15 See e.g. Wüthrich (2013).

16 Gödel (1949).
} 
since (for technical reasons- to be discussed below) it would have to be a contingent affair in GR, there can exist no such objective tensed properties tout court.

In this paper, we appraise critically all such arguments. In brief: we find that the first argument is no good (Sect. 3); the second is most effective against the claim that the actual world is hospitable to objective tensed facts, rather than against the claim that GR in its entirety is not hospitable to objective tensed facts-and A-theorists in the GR context must say more in order to rebut the former of these concerns (Sect. 4); the third has some force-though must be defended against well-known responses due to Swinburne (Sect. 5); and that the fourth invites a range of responses, each of which is worthy of detailed consideration (Sect. 6). Our overall aim is to argue-with greater systematicity and depth than has hitherto been achieved - that although the A-theory certainly has better prospects in GR as compared with SR, more remains to be done by A-theorists in order to respond adequately to the above roster of objections. Moreover, even these issues notwithstanding, the A-theory in the relativistic context only fares as well as in the classical philosophical debates on these matters: if the A-theory fails for general philosophical reasons, relativity theory will not save it. As we will elaborate over the course of this article, our own ultimate view on the issue is that there does not exist sufficient motivation to be an A-theorist in GR.

\section{Cosmic simultaneity}

Before assessing arguments against the use of cosmological solutions of GR in order to ground objective A-theoretic facts, we must present in more detail this A-theoretic programme itself. In this section, we do just this, by focussing on the writings of one particular prominent A-theorist who has advanced such claims: Richard Swinburne.

Swinburne has devoted several writings to the issue of time which cover a broad range of philosophical and theological issues. ${ }^{17}$ He consistently stands with Newton in postulating the existence of absolute simultaneity, arguing that this notion is not necessarily contradicted by relativity theory. He acknowledges the difficulties that the relativity of simultaneity presents for the empirical ascertainability of objective time in SR-however, he maintains that the situation is improved by GR, since cosmological solutions of that latter theory would seem to come equipped with a privileged notion of simultaneity: what he dubs cosmic simultaneity. On this matter, Swinburne writes:

I argue in this paper that Einstein gave good, but not compelling, grounds for supposing that the concept of absolute simultaneity would have no application in a universe empty of matter and so governed entirely by the laws of Special Relativity. But I claim that there is absolute simultaneity in our homogeneous and isotropic universe of galaxies receding from each other with a metric described by the Robertson-Walker line element. 'Cosmic Time' provides a correct standard of absolute simultaneity. ${ }^{18}$

\footnotetext{
17 For example, Swinburne (1968, 1993, 1996).

18 Swinburne (2008, p. 244) (emphasis added).
} 
Swinburne claims that 'cosmic time' provides a standard for absolute time, and that this temporal interval is ascertainable empirically, given the Robertson-Walker line element, in the class of Friedman-Lemaitre-Robertson-Walker (henceforth FLRW) cosmological solutions of GR. ${ }^{19}$ These comprise a handful of the multifarious possible solutions of Einstein's field equations, which in turn represent a certain class of worlds compatible with the laws of GR.

FLRW solutions of GR satisfy the properties of (1) homogeneity (the universe is the same at every point) (2) isotropy (there is no preferred direction in the universe), and (3) expansion (the overall size of the universe evolves dynamically). Imposing these symmetries implies that these models can be foliated by (globally extended) instants, leading to Swinburne's well-defined notion of cosmic time. In turn, this seems to recover the Newtonian picture of instants of absolute space (sometimes: 'spacelike hypersurfaces'), strung together by absolute time. ${ }^{20}$

In light of the notion of cosmic simultaneity which these solutions manifest, they would once again (i.e., harking back to Newtonian mechanics) seem to constitute a hospitable environment for objective tense. As Callender and McCoy write, "the idea that the present is special, that it is what exists or what becomes, is famously threatened by relativity. Finding a scientifically respectable absolute time according to which the world comes into being would give [the A-theorist] "proof" that they need." 1

We agree with these authors: thus, in our view such arguments mustered by Atheorists like Swinburne warrant serious engagement. To this end, in the following four sections of this article we evaluate four central arguments which have been levied against the use of cosmic time to ground an objective past/present/future distinction in GR.

\section{Changing the theory?}

That cosmological solutions of GR seem to afford an hospitable environment for an objective past/present/future distinction has been known since shortly after the construction of those solutions. For example, James Jeans wrote in 1936 that $^{22}$

Now, the second property [in addition to expansion] which all the mathematical [cosmological] solutions have in common is that every one of them makes a real distinction between space and time. This gives us every justification for reverting to our old intuitional belief that past, present and future have real objective meanings, and are not mere hallucinations of our individual minds ... [W]e find a distinction between time and space, as soon as we abandon local physics and call the astronomy of the universe to our aid.

\footnotetext{
19 Smeenk (2013, p. 204).

${ }^{20}$ For a more technical discussion of this material, see e.g. Callender and McCoy (forthcoming), or Smeenk (2013). The above presentation will suffice for our purposes.

${ }^{21}$ Callender and McCoy (forthcoming, p. 4). See also Callender (2017, ch. 3). We take the present paper to be complementary to that piece.

22 Jeans (1936).
} 
In a recent paper, however, Monton is critical of all such claims, maintaining that to focus thus on cosmological solutions of GR is, in fact, to change the theory under consideration. To be more specific, Monton writes, ${ }^{23}$

Granted, such a foliation can sometimes be added to the spacetime: for some models of general relativity, for example, the spacetime structure itself allows one to pick out a foliation ... . But the point is that the foliation is not a part of the spacetime structure as given, and thus imposing such a foliation amounts to changing the theory.

What to make of this critique? Wüthrich responds to Monton as follows: ${ }^{24}$

It is somewhat mysterious why he [Monton] has such qualms about changing the theory, particularly since at the end of his essay, he has no hesitation to proclaim that a committed presentist ought to demand that since string theory and loop quantum gravity do not account for presentist intuitions, they ought to be modified accordingly.

Wüthrich's focus in the above quote is upon presentism - though for our purposes we can read him as making a statement about any tensed view of time. His point, then, is that it is puzzling that Monton objects to changing GR in order to accommodate objective tense, when he is quite willing to do so in the context of quantum theories of gravity, such as string theory and loop quantum gravity. ${ }^{25}$

Does this critique of Monton hold up? Arguably, it is not fair: for nowhere in his paper does Monton object to modifying GR in order to accommodate objective tense; rather, he makes the conditional claim that if one wants to reconcile objective tense with GR, then the latter will (in fact) have to be modified. This is consistent with the parallel claims which Monton makes with respect to certain contemporary theories of quantum gravity; thus, there is no puzzling inconsistency in Monton's views.

In any case, in our view Wüthrich's critique of Monton concedes too much-for to focus on the structures in place in a particular class of solutions of GR is not to change the theory. Rather, the A-theorist in the context of GR only faces the charge of changing the theory if she attempts to incorporate the structures present in the particular solutions in GR under consideration (in particular in this case, cosmic simultaneity) into all possible solutions - that is, if she uses this structure contingent in GR to revise her space of kinematical possibilities, such that all such possibilities manifest this structure. (One way to do this would be to move from GR to an alternative- but empirically equivalent - theory known as 'shape dynamics', which does feature objective simultaneity in all solutions. ${ }^{26}$ Another would be to resort to Swinburne's 'flat-footed' response, discussed in Sect. 6.2 below.) This, however, is transparently not what most advocates of tense in GR are seeking to do-and thus, Monton's objection here cuts no ice.

\footnotetext{
23 Monton (2006, pp. 267-268).

24 Wüthrich (2010).

25 The details of these theories do not matter for our purposes, but see e.g. Huggett and Wüthrich (2013), for a philosophical introduction.

26 See e.g. Mercati (2018).
} 
A related concern which one might have in this vicinity is the following: absent cosmic simultaneity being 'baked into' the kinematics of one's theory (as, at least for some, in e.g. Newtonian mechanics ${ }^{27}$ ), is this dynamically selected simultaneity structure, present in only some solutions of the theory, sufficient to ground objective A-theoretic facts? We see no reason why the answer here cannot be positive. Indeed, our answer in this regard would mirror exactly that given by Brown (an author well-known for repudiating the kinematics/dynamics distinction) in the context of Newtonian mechanics. In that theory, Brown's argument is that simultaneity is just as conventional as in e.g. $\mathrm{SR}^{28}$ — for which events one regards as being simultaneous is simply a matter of how one "spreads time through space"-i.e., sets up temporal coordinates. Brown writes: ${ }^{29}$

Newton spread time through space in inertial frames in such a way that actionsat-a-distance like gravity are instantaneous and do not travel backwards in time in some directions. It is a highly natural convention-it would be barmy to choose any other-but it is a convention nonetheless, and one consistent with the standard Galilean coordinate transformations.

Brown's claim is that, in Newtonian mechanics, a preferred foliation into hypersurfaces of constant time falls out naturally from dynamical considerations: it is said foliation which simplifies the physics. We make exactly the same claim vis-à-vis cosmic simultaneity in the context of GR - and thus, insofar as one can use this privileged simultaneity structure in Newtonian mechanics to ground an objective past/present/future distinction, one may make the exactly analogous move in the cosmological context.

These issues are touched upon also by Callender and McCoy, who write: ${ }^{30}$

There is a natural standard of time to use in a non-vacuum FRW spacetime: the proper time of anything not moving with respect to the cosmic fluid. Hypothetical objects that are at rest with respect to it are called fundamental observers. Galaxies, for example, are approximately fundamental observers, since they do not move much with respect to the background fluid. The proper time of the cosmic fluid, which we will denote by $t$, is generally known as cosmic time.

Nevertheless, although the decomposition from above naturally picks out a particular time function $(t)$, the theory is still entirely relativistic. We chose to foliate spacetime into slices of constant spatial curvature, but we could just as well have chosen an infinity of other foliations. It is therefore only when we choose the natural foliation and use cosmic time that one can claim that the universe is 13.8 billion years old - this duration is determined by the proper time of a hypothetical fundamental observer from the beginning of time until now. By contrast, to

\footnotetext{
27 See e.g. Malament (2012, ch. 4).

28 For an admirably clear presentation of the philosophical issues surrounding the conventionality of simultaneity in relativity theory, see Janis (2018).

29 Brown (2005, p. 20).

30 Callender and McCoy (forthcoming).
} 
photons the world just began. Relativity doesn't choose sides over who is right, fundamental observers, arbitrarily moving observers, or photons. All are.

Again, we agree with these authors on this point: although a range of foliations are still possible in cosmological solutions of general relativity, there is, nevertheless, a uniquely dynamically privileged such foliation: again, that which maximally simplifies one's physics.

Still, one might reasonably question: why should what is dynamically privileged be metaphysically privileged? We agree that this remains an open question, and that further argumentation is necessary to fully bridge this gap. Such argumentation could mirror Maudlin's observation that the principle of sufficient reason would allow Leibniz to avoid the force of the kinematic shift in Newtonian mechanics, since there does exist a dynamically privileged element of the class of kinematically-shifted Newtonian worlds: viz., that with zero velocity with respect to the persisting points of absolute space-so God would have reason to actualise this solution rather than any other. ${ }^{31}$ But in any case: the A-theorist should be explicit about why and how tensed properties are being piggybacked upon a dynamically privileged structure. ${ }^{32}$ Of course, the same charge could be levied against Brown-indeed, we take it that such is a very natural objection to Brown's position, which may be advanced by advocates of the opposing 'geometrical perspective' (as situated against Brown's 'dynamical perspective' on spacetime theories). ${ }^{33}$ To bridge this gap, Brown must respond with some kind of metaphysical thesis - as indeed he does, in his advocation of a programme of ontological reduction of spacetime structure to dynamical symmetries. ${ }^{34}$

To close our discussion of these matters, it is helpful to visualise the solution spaces of Newtonian mechanics versus SR versus GR. In Newtonian mechanics, every solution of the theory has a dynamically preferred simultaneity surface (as discussed above). In SR, by contrast, in light of the relativity of simultaneity, while spacetime is foliable into hypersurfaces, there is no dynamically privileged such hypersurface - and this holds, again, for all solutions of the theory (since all solutions manifest the structure of Minkowski spacetime). The situation in GR is more complex, and the solution space of this theory partitions into three classes. First, there are solutions of the theory which do not admit any foliation into hypersurfaces. (The desire to illustrate this point is, indeed, what first led Gödel to construct his famous time-travel solution of GR. We return to Gödel below. ${ }^{35}$ ) Second, there are solutions of GR which admit no privileged foliation (naturally, Minkowski spacetime falls into this class). Third, there are (it would seem!) solutions of GR, such as the cosmological solutions already discussed, which admit of such a privileged foliation. The situation here is summarised in Fig. 1; the claim to be investigated is that the simultaneity structure present in this third class of solutions can constitute the basis on which to ground an objective past/present/future distinction in GR.

\footnotetext{
31 Maudlin (1993, pp. 191-192).

32 Our thanks to Niels Martens for discussion on this point.

33 For a book-length defence of the dynamical perspective, see Brown (2005). For a recent defence of the geometrical perspective, see Maudlin (2012).

34 Brown and Pooley (2006).

35 Gödel (1949).
} 

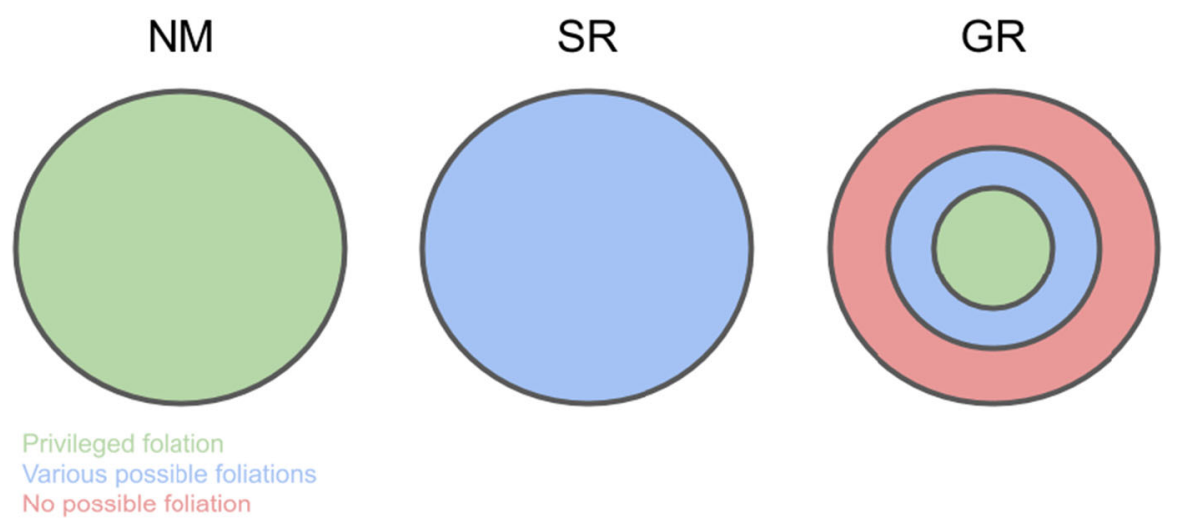

Fig. 1 The solution spaces of Newtonian mechanics (NM) versus special relativity (SR) versus general relativity (GR), as regards the possibility of spacetime foliations within the solutions of those theories

\section{Modelling and averaging}

We turn now to what is, in our view, a more promising line of argument against the place for objective tense in general relativity. Here is how Smeenk puts the concern: ${ }^{36}$

Questions like those mentioned in the introduction-"how old is the universe?"- are only well-posed within a remarkably restricted class of spacetime models. The questions are moored to the structure of the models fixed by strong symmetry assumptions, so to speak. But in more realistic models describing a lumpy, bumpy "almost-FLRW" universe, these questions are cast adrift.

In light of their manifesting the properties of absolute homogeneity and isotropy, FLRW solutions of GR are highly idealised-so one might ask (following e.g. Nor$\operatorname{ton}^{37}$ ): can our world really be an FLRW universe? The answer, clearly, is no. But in that case, the question in turn arises: in an 'almost-FLRW' universe such as our own, do we still have available a well-defined notion of cosmic simultaneity? If not, then there would seem to exist serious problems for the A-theoretic project of grounding objective tense.

When responding to this point from Smeenk, it is important to distinguish two questions:

1. Is an objective past/present/future distinction possible in GR?

2. Is an objective past/present/future distinction possible in the GR solution corresponding to the actual world?

Smeenk's concerns speak only to the second point here-insofar as they lead us to question whether one can ground an objective past/present/future distinction in the actual world. Transparently, this is distinct from the former question-which regards the possibility of grounding such a distinction in at least one solution of GR. Since

\footnotetext{
36 Smeenk (2013, p. 7).

37 Norton (2012).
} 
Smeenk concedes (as he should) that the FLRW solution of GR is, indeed, a legitimate solution, the above concerns do nothing to lead one to question invoking an affirmative response to (1). The moral here, then, is that one has to be clear on the nature of one's project: in the remainder of this section, we focus on addressing Smeenk's negative answer to the second of the above two questions.

Whether a privileged notion of cosmic simultaneity is available in the actual world is an open empirical question, the answer to which depends upon (a) our gathering sufficient empirical data to determine which specific perturbed-FLRW solution corresponds to our own world, and upon (b) theoretical appraisal of whether that solution admits of cosmic simultaneity - that is, whether that solution retains a dynamically privileged simultaneity choice. Clearly, any affirmative answer to (2) (such as that given by Swinburne) must wait upon answers from physics to (a) and (b). In the meantime, however, any such claim can only be conditional in nature.

\section{Temporal experience}

The previous section was concerned primarily with whether an A-series is vindicated by, or at least compatible with, GR. It is important, however, to ascertain whether the proposed structure upon which the split between past and future is grounded can connect with our temporal experience-for it is often just that experience which motivates the A-theorist to hold her position in the first place. As Huw Price writes,

In practice, the most influential argument in favour of the objective present and the objective flow of time rests on an appeal to psychology - to our own experience of time. It seems to us that time flows, the argument runs, and surely the most reasonable explanation of this is that there is some genuine movement of time which we experience, or in which we partake. ${ }^{38}$

Temporal passage is phenomenologically immediate: we anticipate the future and remember the past, and this structure is an integral feature of our psychological existence. Though Price goes on to argue that "arguments of this kind need to be treated with caution," 39 he is right in noting the power of temporal experience in motivating A-theoretic standpoints. With this in mind, it is fair to assume that one central reason for which A-theorists seek to introduce tensed properties into GR is to underwrite this phenomenological conception of temporal passage. Therefore, it is of critical concern that we establish whether the kind of tensed properties cosmic simultaneity could allegedly establish are recognisable in the context of our experience. If not, then the reason for which those properties were introduced is undermined.

Several authors in the recent literature have maintained that cosmic simultaneity is not up to the task of grounding our experience of temporal passage. For example, Wüthrich writes: ${ }^{40}$

\footnotetext{
38 Price (1996, pp. 14-15).

39 Price (1996, pp. 14-15).

40 Wüthrich (2013, p. 17).
} 
In all this, it remains utterly mysterious how this highly idealized construction connects to our intuitions regarding temporal becoming and the present. If the absolute time constructed from this idealizing averaging procedure over vast cosmic scales is the time which determines what is the present, then how does the human perceptual and cognitive apparatus latch on to this idealized structure?

In his monograph, Bourne echoes such sentiments: ${ }^{41}$

... [T] he notion of simultaneity under this definition is so divorced from our experience that it leaves us far removed from our intuitions about the notion of temporal becoming, and what it is for events - that is, everyday events - to be simultaneous.

Once again, in assessing whether these concerns raised by authors such as Wüthrich and Bourne are legitimate, it is apposite to distinguish two questions:

1. Is an objective past/present/future distinction possible in GR?

2. How would the structure upon which that distinction is grounded connected with our phenomenological temporal experience?

Clearly, nothing in this particular objection calls into question whether grounding Atheoretic properties in a GR universe is coherent-rather, the issue at stake is whether it is sufficiently well-motivated. It is, accordingly, upon this latter concern that we focus in the remainder of this subsection.

The apparent problem regarding the disconnect between cosmic simultaneity and our temporal experience is the following. The cosmic scales with which we are dealing when discussing cosmic time-viz., scales of the entire universe-are extremely far removed from our quotidian experience in our world of medium-sized dry goods. More specifically: our temporal experience is, plausibly, a function only of our local environment, rather than of the entire universe. Absent argumentation to the effect that we can, in fact, have local access to cosmic time, it is not at all obvious that these cosmological notions can do the work of grounding our local temporal experience, as the A-theorist desires.

Indeed, there are good prima facie grounds to question whether this gap can be bridged. Local observers in a relativistic universe will be subject, in principle, to all familiar relativistic effects: such as time dilation, gravitational redshift, etc. In light of this, it is reasonable to ask: how could any such observers account for all such effects, and thereby establish a reliable means of gaining access to cosmic time? As Smeenk puts the matter, "a globally defined cosmic time function generally doesn't correspond to the time elapsed on an observer's wristwatch."42

Swinburne has sought to address these concerns in the following way. ${ }^{43}$ First, recall from the quotation from Callender and McCoy presented in Sect. 3 that galaxies are approximate 'fundamental observers' - i.e., physical bodies such that any ideal clock comoving with those bodies would read off intervals of cosmic time. ${ }^{44}$ Given that

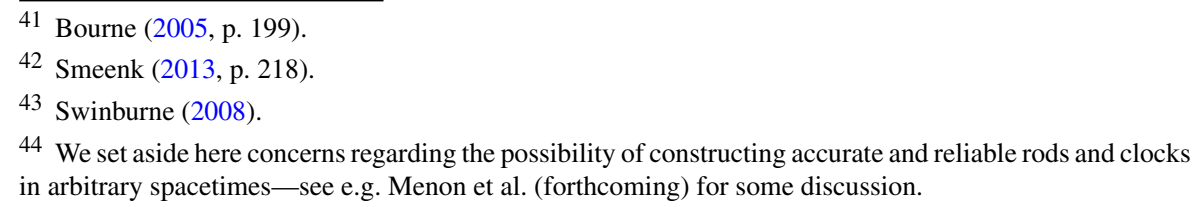


human agents are observers within one such galaxy, Swinburne claims that we, too, can have approximate access to cosmic time. If that is correct, then the gap between cosmic time and our local temporal experience is bridged. ${ }^{45}$

Unfortunately for Swinburne, more needs to be done in order to make this response convincing - the reasons for this run along three distinct axes. First, in spite of our being situated in approximately fundamental observers (galaxies), we ourselves are not fundamental observers. As Pettini writes, ${ }^{46}$

Are we fundamental observers? No, because:

- The Earth rotates around its axis and orbits the Sun.

- The Sun moves relative to the Local Standard of Rest (the local reference frame defined by the motions of the stars nearest the Sun).

- The Sun is one of $\sim 10^{11}$ stars in a spiral galaxy, the Milky Way.

- The LSR orbits the centre of the Milky Way Galaxy with a period $\mathrm{P} \simeq 240$ million years.

- The Milky Way moves relative to the centre of mass of the Local Group.

- The Local Group is infalling into the Virgo Cluster of galaxies.

- The Virgo Cluster, along with several other large clusters, is in turn speeding towards a great concentration of mass known as 'The Great Attractor'.

All of these considerations, coupled with the fact that (to repeat) there may exist further relativistic effects within our galaxy (e.g. gravitational redshift) certainly means that more needs to be done in order to make compelling the case that we can have local access to cosmic time. Second: there is the concern that galaxies (or, if one prefers in light of the above quotation, galactic superclusters, etc.) are only approximate fundamental observers in our universe - so even accessing their associated 'fundamental frames' would not fully bridge the gap between our local experience and cosmic time. And third: one may simply find it unbelievable that whether I regard (for example) two events as being simultaneous must depend upon properties of the entire universe-in which case, even if Swinburne can overcome the technical issues discussed above, some may nevertheless resist any appeal to cosmic time as a means of grounding agential temporal experience. ${ }^{47}$

Our conclusions on these matters are, then, the following: while one motivation to introduce objective tense is to account for our phenomenological experience of temporal passage, associating such objective tensed facts with cosmic simultaneity surfaces in a GR universe does not obviously successfully achieve this goal, given that

\footnotetext{
45 It is worth registering that Swinburne deploys the above arguments to a slightly different end in his article: that is, in order to demonstrate that cosmic time is operationally ascertainable in principle, and so must be meaningful. We agree with this claim-if emphasis is placed upon 'in principle'. However, in practice, there remains a gap between cosmic time and our temporal experience, in light of the issues presented above. Moreover, even if this gap can be bridged, those with B-theoretic predilections might still resist the use of cosmic simultaneity to ground objective tense.

46 Pettini (2018, pp. 1-2).

47 Note that this concern would also apply to any attempt to ground temporal experience in Newtonian mechanics in the privileged simultaneity structure available in that theory-at least, if Newtonian mechanics is understood in the four-dimensional framework of e.g. Malament (2012, ch. 4). Our thanks to Oliver Pooley for discussion on this point.
} 
such cosmic simultaneity surfaces appear to be, in an important sense, disconnected from, and inaccessible to, us qua finite observers. While it is possible that work can be done to bridge this gap, more needs to be said by the A-theorist to realise this goal. Otherwise, this particular A-theoretic motivation to introduce object tense, and tie it to cosmic simultaneity surfaces, is uncompelling.

\section{Gödel's modal moral}

In 1949, Gödel presented a novel, modal argument against the introduction of primitive tensed properties into (cosmological solutions of) GR. He wrote: ${ }^{48}$

... if someone asserts that this absolute time is lapsing, he accepts as a consequence that, whether or not an objective lapse of time exists ... depends on the particular way in which matter and its motion are arranged in the world. This is not a straightforward contradiction; nevertheless, a philosophical view leading to such consequences can hardly be considered as satisfactory.

Gödel's claim here is that it is metaphysically unacceptable to introduce primitive tensed properties into some, but not all, solutions of GR-for such properties are not, he claims, the kinds of things which can be contingent: either they exist in all possible worlds, or they exist in none. ${ }^{49}$

Gödel's argument is subtle, and requires unpacking before a full appraisal is possible. We will do so over the course of the following subsections. In Sect. 6.1, we present Belot's claim that the above argument can be evaded via a tactic which he dubs "monster-barring". In Sect. 6.2, we consider a potential flat-footed response to Gödel's argument. In Sect. 6.3, we consider whether Gödel's argument is truly modal in nature-or whether, rather, Gödel is instead proposing a norm on interpretative good practice. In Sect. 6.4, we consider a response to Gödel's argument which has come to be known as "Earman's challenge", and assess two ways to implement this: (1) the introduction of primitive tensed properties, and (2) functionalism about tense. In the latter case, we draw connections with Maudlin's approach to the flow of time in physical theories.

\subsection{Evasion by monster-barring}

Gordon Belot proposes a simple tactic to mitigate the force of Gödel's argument: impose extra conditions on one's theory, in order to restrict the allowed class of solutions (here of GR) to only (a subset of) the solutions which admit of a privileged foliation (i.e., the green solutions in the space of solutions of GR presented in Fig. 1); this he dubs "evasion by monster-barring". 50 There are, indeed, various restrictions

\footnotetext{
48 Gödel (1949).

49 Indeed, as mentioned above, Gödel's original motivation for constructing his famous time travel solution of GR was to show that there are some models of that theory which cannot be foliated into spacelike hypersurfaces at all - thereby allowing him to make the above philosophical point.

50 Belot (2005).
} 
which are often imposed by practicing general relativists when working with the solution space of this theory-for example, one may focus only on solutions of the theory which can be expressed in the Hamiltonian formalism (since it is such solutions which are taken to admit more readily of quantisation), ${ }^{51}$ or one may focus on solutions of the theory which satisfy certain energy conditions or causality conditions. ${ }^{52}$

Let us suppose that such restrictions $d o$ in fact whittle the class of solutions of GR to only those involving a privileged foliation. If this were the case, then there is a sense in which Gödel's argument would be avoided immediately, for now primitive tensed properties could be introduced in all solutions-thus rendering them non-contingent, in line with Gödel's requirements. In practice, however, it is hard to be convinced by this response-for, plausibly, to introduce such auxiliary conditions (which can be understood as extra dynamical equations) is precisely to change the theory: cf. Monton's point, discussed in Sect. 3. Given this point, we will henceforth set aside this particular tactic for responding to Gödel, and will restrict ourselves to consideration as to whether Gödel's concern can be evaded within the context of GR.

\subsection{A flat-footed response}

Recall that, for Swinburne, while some concepts - such as that of time-feature operationalisability in their very definition, others — such as simultaneity — do not. That this is so is evident from passages such as the following: ${ }^{53}$ (Here, by "primary test", Swinburne means some operational test for ascertaining the value of a given quantity.)

Now there are, I think, grounds for supposing that our concept of (absolute) simultaneity is more basic than the primary tests for its application; whereas by contrast, I claimed that there was no more to the concept of equal temporal interval at a place than was contained in the tests for its application.

Since Swinburne does not think that simultaneity must be determinable operationally in order to be meaningful, there is room for him to simply deny the conclusion of Gödel's argument: a privileged (albeit super-empirical) notion of simultaneity does exist in all solutions of the theory-including e.g. the Gödel time-travel solution. The idea is that one interprets all solutions of GR such that there also exists extra absolute simultaneity structure, which is decoupled from the matter content of the universe, and so which is not empirically detectable. Thus, for Swinburne, all solutions of GR presented in Fig. 1 should, in fact, be coloured green.

We concede that this is a viable response to Gödel—albeit one which may seem under-motivated to one without prior reasons to favour an A-theoretic conception of time, or to one with naturalistic predilections in philosophy. ${ }^{54}$ For the sake of thoroughness, though, it is worth emphasising some further aspects of the view. In particular,

\footnotetext{
51 These are the conditions upon which Belot himself focuses-see Belot (2005, p. 275).

52 For a virtuoso survey of the former, see Curiel (2017).

53 Swinburne (2008, p. 254).

54 Swinburne has confirmed to us in personal communication that he would endorse the above-articulated line of reasoning. Clearly, this tactic is one natural possible generalisation of the neo-Lorentzianism of e.g. Lane Craig to the context of GR.
} 
one might ask: how is the privileged simultaneity structure present in all solutions of one's theory (here GR) to be implemented? Is one to envisage extra (e.g. Newtonian) spacetime structure, which is decoupled from the dynamics (which adverts only to the relativistic spacetime structure) but which is nevertheless present in all solutions of the theory? Such a picture would certainly suffice as a means of implementing the suggestion; however, some perplexing consequences would follow. First, if the relativistic dynamics is decoupled from the simultaneity structure in some solutions, surely this would also apply in e.g. FLRW solutions? And in that case, the fact that cosmic simultaneity coincides with this extra postulated absolute simultaneity structure would seem miraculous. While one response here might be to declare that, in all solutions, the absolute simultaneity structure just is that of an FLRW spacetime (so that one does not face the above-described question of miraculous coincidence in those FLRW universes), this would seem to be radically anti-empirical: the upshot would be that every GR world is an expanding, big bang-type universe-but in the majority of such worlds, no empirical data provides any evidence to think that is the case. In addition: a second perplexing consequence of this view would be that, in e.g. Gödel time-travel universes, two events which are regarded as being timelike-separated according to the physical, relativistic spacetime structure may nevertheless be regarded as simultaneous according to the 'metaphysically prior' simultaneity structure. ${ }^{55}$ While authors such as Swinburne may simply accept this consequence, it should be stressed that this is a stronger claim than one encounters when considering e.g. neo-Lorentizanism in $\mathrm{SR}$, according to which only spacelike-separated events stand in the primordial simultaneity relation. Indeed, for many, to speak of timelike-separated simultaneous events will be to push the notion of simultaneity to breaking point. ${ }^{56}$

\subsection{Metaphysics or interpretation?}

One might be perplexed by Gödel's argument, on the following grounds. Consider a world associated with a given solution of a given theory (say, GR). While it is plausible that the mathematics of that solution fixes the physical facts, ${ }^{57}$ it is also plausible (at least to us!) that said mathematics underdetermines the metaphysical facts. If that is correct, then there will, in fact, be multiple possible worlds associated with a given solution of a given theory. ${ }^{58}$ For example: there might be one general relativistic world in which spacetime points have primitive identities, and another in which they do not; or-more relevant to our purposes - there might be one cosmological general

\footnotetext{
55 For the technical details of Gödel spacetimes, see e.g. Malament (2012, ch. 3).

56 Our thanks to Martin Pickup and Oliver Pooley, and to an anonymous referee, for discussions on these matters.

57 Of course, a neo-Lorentizan of the kind considered in the previous subsection would dispute this.

58 This view is known as 'metaphysical contingentism'; although historically a minority view, it has found recent defence in the work of, for example, Rosen (2006) and Miller (2009). Le Bihan (2014) argues that the existence of Gödel solutions of GR shows that 'no-futurism' (the view that the past and present are real, but the future is not: when combined with A-theoretic passage, this yields the growing block view) would have to be metaphysically contingent_-and so, following a line of reasoning similar to that of Gödel, should be rejected.
} 
relativistic world in which events are endowed with primitive tensed properties, and another in which they are not.

If the foregoing is correct, then Gödel's argument would seem to be unduly strong, for it would preclude by fiat that said metaphysical underdetermination is possible - for its being possible would render all metaphysical properties contingent - in which case Gödel's argument would seem to yield the result that no metaphysical properties, objects, etc., can exist in any possible world (for if they were to exist in some worlds, then they would not exist in others, by the contingency thesis-thereby violating Gödel's argument). ${ }^{59}$ In other words, therefore, Gödel can be read here as claiming that physical objects and quantities have their metaphysical properties of necessity: an historically mainstream thesis to be sure, but one which seems (to us!) to be at once overly strong and under-motivated. ${ }^{60}$

In light of this, is there an alternative way to understand Gödel's argument? We would like to suggest here an alternative reading, according to which Gödel would not deny the above-described metaphysical underdetermination, but according to which, given said underdetermination, Gödel proposes a certain norm on interpretative good practice vis-à-vis our physical theories.

The thought is the following. Given that metaphysical facts are (speaking loosely) supposed, in some sense, to be prior to physical facts, it would be ill-motivated to interpret the solutions of one's physical theories such that some of the associated worlds are endowed with certain metaphysical properties, and other worlds with other properties. (Such an approach would be in tension with the mainstream line that, once the metaphysical possibilities are fixed, the physical possibilities should be a subset thereof.) Thus, one should interpret all solutions of one's physical theories so as to manifest the same metaphysics - whether (to focus on the example pertinent to our case) that be A-theoretic metaphysics, or B-theoretic metaphysics, or whatever.

Given this reading, Gödel's argument is more pertinent, for in GR it would seem (in light of the heterogeneous structure of its solution space as illustrated in Fig. 1) that such interpretative uniformity is not possible when it comes to objective tense: at most some solutions of the theory can be interpreted such that the associated worlds manifest primitive tensed properties. But in that case, it is better-on grounds of interpretative hygiene — not to introduce such tensed properties at all. In our view, this is certainly a more defensible reading of Gödel on this issue. However, responses on behalf of the A-theorist are still possible. First, an A-theorist may have sufficiently strong alternative philosophical motivations to introduce, in a contingent manner, certain metaphysical properties into one's interpretation of a given theory in order to override the above interpretative concern. Second, there is, perhaps, room for such Atheorist to maintain in fact that they are following uniform interpretative norms-for example, of the form: 'Introduce A-theoretic properties whenever one can; otherwise, do not.' Third, an A-theorist might follow the non-naturalistic strategy articulated in

\footnotetext{
59 'Ontic structural realists' motivate the excision of metaphysics by way of its apparent ability to overcome metaphysical underdetermination-see French (2011). For some critical remarks on ontic structural realism, see Pooley (2006). The potential epistemological objections to said metaphysical underdetermination propounded by Le Bihan (2014) are reminiscent of what, in the literature on symmetries in physics, is known as an 'Occamist norm' to undetectable, symmetry-variant structure: see Dasgupta (2016).

60 Our thanks to Martin Pickup for discussion on this issue.
} 
the previous subsection. Thus, in sum, while this version of Gödel's argument might have some force for some authors, and is interestingly different to the standard reading of Gödel in itself, it is unlikely to convince anyone who, for other reasons, is already an A-theorist in the context of cosmological solutions of GR.

\subsection{Earman's challenge}

John Earman tackles Gödel's argument head-on. He writes: ${ }^{61}$

There seems to be no lurking contradiction or anything philosophically unsatisfactory in saying in the same breath: "Space in the actual world is open, but if the mass density were a little greater, space would be closed," or "Time in the actual universe goes on forever into the future, but if the mass density were greater the universe would eventually recollapse and time would come to an end." Why then is there a lurking contradiction or something philosophically unsatisfactory in saying: "Time in our universe lapses, but if the distribution and motion of matter were different, there would be no consistent time order and so time would not lapse"?

Belot dubs this Earman's challenge. ${ }^{62}$ The challenge is a simple one: deny Gödel's intuition that all worlds associated with a given theory must be such that they manifest the same metaphysical properties. While on the face of it, this may seem ad hoc-for example, Bourne asks, ${ }^{63}$

$\ldots$ is it plausible that the metaphysical notion of temporal becoming can be equated with a particular physical process that is contingent on the particular distribution of matter in the universe?

- we have already seen in the previous subsections reasoning which might motivate this move.

On the other hand, in place of introducing primitive tensed properties into our interpretation of given physical theories, is there any other means by which Earman's challenge could be implemented? An alternative in this regard has recently been proposed by Bourne: that we should be functionalists about tensed properties. He writes: ${ }^{64}$

A much better proposal [than the invocation of primitive tensed properties] is this: the only appealing way of viewing the matter is to invoke the notion of a functional role: specifically, we might hold that cosmic time plays the temporalbecoming-role in worlds where cosmic time is available, whereas other processes in another world might play the temporal-becoming-role in that world.

Recall that, for a functionalist, 'to be $X$ is to play the $X$-role'. (The most famous example of functionalism can be found in the philosophy of mind: the functional role of pain is to cause agents to withdraw from harmful circumstances; this role is played

\footnotetext{
61 Earman (1995, p. 198).

62 Belot (2005, p. 271).

63 Bourne (2010, p. 201).

64 Bourne (2010, pp. 201-202).
} 
by the firing of C-fibres in our nervous systems; thus, pain just is the firing of C-fibres.) Thus, the idea of functionalism about tense is that we can identify some structure in a fundamentally B-theoretic world, which nevertheless plays the functional role of tense. $^{65}$

The problem with this proposal is that it is not at all transparent what such structure could be. Indeed, prima facie there appears to be nothing in a B-theoretic world which plays the role of changing over time à la the A-theorist's moving present. Thus, in practice, it is not clear how this approach to tense-intended as a response to Gödel's modal argument - can succeed. In places, Bourne does appear to acknowledge this issue-for example, he writes: 66

On discovery that the universe is not a steady-state universe, for example, rightthinking people don't start wondering whether the world might be tensed in light of this. ... Thus it is not at all clear that cosmic time can be identified with, or play the role of, temporal becoming as it is conceived of in this world.

The concern here is on-point-although it is articulated (in our view) for the wrong reasons. The issue is not that people do not doubt the existence of tensed properties even in universes where the evidence suggests that such properties cannot exist. ${ }^{67}$ Rather, the issue is that in no general relativistic world, interpreted without primitive A-theoretic facts, does there appear to be anything which can play the functional role of those tensed properties.

There are connections here with Tim Maudlin's views on the philosophy of time. Although Maudlin maintains that the flow of time is a genuine feature of reality, he also maintains that the only fundamental ontology that he need commit himself to is that of the B-theorist. ${ }^{68}$ How, then, is the flow of time supposed to be recovered from this ontological picture? Maudlin writes the following: ${ }^{69}$

The theory of time's passage I defend focuses on the B series: all events ordered by a transitive, asymmetrical relation of earlier and later. Given events ordered in a B series, one can define an infinitude of different A series that correspond to taking different events as 'now' or 'present'.

Maudlin claims that he can recover an infinity of A-series from a given B-theoretical description of the world: presumably, one such A-series for each particular (cosmic_though see Sect. 7 below) time being present. While this is correct, it is inadequate as a means of recovering a unique, objective flow of time, given the

\footnotetext{
65 Functionalism is currently à la mode in the philosophy of physics: for other examples, see functionalism about classical worlds (Wallace 2012), functionalism about spacetime (Knox 2019), and functionalism about gravitational energy (Read forthcoming).

66 Bourne (2010) A future for presentism, p. 202.

67 Though, again, cf. Sect. 6.2.

68 This view is similar to that of Oaklander, who advocates (following a certain reading of Russell, hence the nomenclature) an 'R-theory' of time, according to which the 'becoming' aspect of time exists already in the primitive orientation of the temporal relations. For details, see Oaklander (2012).

69 Maudlin (2007, ch. 4, p. 126. fn. 11).
} 
multiple-realisability which Maudlin acknowledges. ${ }^{70}$ In other words, Maudlin's points here seem to confirm the view that there is not sufficient structure in a GR universe to uniquely play the functional role of tense. But given that uniqueness is what the A-theorist is after, it is not clear how Bourne's functionalism about tense can succeed.

One might think that the functionalist can simply make an arbitrary choice here as to which A-series is the true, physical A-series. However, in a straightforward sense this move is incompatible with functionalist motivations-for, as we have seen, there is nothing in the physics which motivates said choice. Thus, it is not clear to us that this is a move which the functionalist about tense should be willing to countenance. ${ }^{71}$

\section{Local tense}

Let us summarise the dialectic up to this point. Although Newtonian mechanics appears to be an hospitable environment for absolute simultaneity - and therefore for objective tense-such is not the case in SR, due to the relativity of simultaneity. In GR, however, the prospects for objective tense would again seem to be improved, due to the existence of dynamically-privileged foliations in certain cosmological solutions of that theory: what Swinburne dubs 'cosmic simultaneity'.

Naturally, such arguments in favour of the recovery of objective tense in these solutions of GR have been subject to scrutiny; in this paper, we have assessed four such arguments against said grounding of objective tense. The first-the accusation due to Monton that to proceed in this manner is to change the theory under consideration-we have found to be misguided. The second - the claim that (e.g.) FLRW universes are mere idealisations to the actual world, we have found to be (1) too quick, given our lack of current knowledge regarding whether cosmic simultaneity is still possible in empirically-relevant perturbed FLRW solutions; and (2) to speak most centrally against the claim that objective tense is possible in the actual world, rather than in any GR world. The third objection-that cosmic time is radically disconnected from our local, agential experience of time - is a concern to which an A-theorist such as Swinburne may be able to respond: though more needs to be done to show how local agents can have access to cosmic time, in order to make such a response fully compelling. The fourth-Gödel's modal argument, invites (a) responses which proceed by 'monster-barring', and (b) non-naturalist responses such as that of Swinburne. Even interpreted as a norm on good interpretative practice, the argument can be evaded, for there are various ways in which an A-theorist can argue that they are being interpretatively consistent, even when interpreting solutions of GR such that only some of the associated worlds as exhibiting primitive tensed properties. While Bourne proposes functionalism as a means of avoiding the introduction of primitive tensed properties, and therefore as a natural means of justifying the existence of tensed properties in some but not all GR worlds, it is not clear how this functionalism is supposed to work.

\footnotetext{
70 Maudlin's view here appears to be roughly analogous to that according to which there are multiple different gravitational energies in GR: one for each frame of reference. See Read (forthcoming) and Pitts (2013).

71 We are grateful to an anonymous reviewer for this suggestion.
} 
Overall, therefore, it appears that an A-theorist may-with some further work-be able to respond to all major objections to the programme of using cosmic simultaneity to ground objective tense in GR. Unequivocally, then, the situation is better for the A-theorist in GR than it is in SR. One should, however, resist at this point drawing the conclusion that we should be A-theorists in the context of GR-for all the arguments against the A-theory which arise in the classical debate will continue to apply in this context. In particular, most philosophers of a certain naturalistic bent (such as Mellor ${ }^{72}$ ) will not find the A-theory palatable in GR any more than in any other theory. In other words, then: relativity theory presents no special reasons in favour of objective tense: whatever arguments one favours for or against this view in the original, classical debates will continue to apply in this context.

Though the above is, essentially, our final take on these matters, we wish to consider in this section one final interesting recent view which has arisen in the contemporary literature. Specifically, we will consider here Maudlin's claim that one does not require a privileged, global notion of simultaneity in order to ground objective tense in GR. Maudlin justifies this claim thus: ${ }^{73}$

... The passage of time provides, in the first instance, a fundamental objective distinction between two temporal directions in time: the direction from any event towards its future and the direction from any event towards its past. If we want to distinguish, for example, an asteroid going from Earth to Mars from an asteroid going from Mars to Earth, what do we need? We may focus completely on the world-line of the asteroid in question. Everyone agrees that one end of the worldline has Earth in the near vicinity of the asteroid, and the other end has Mars in its near vicinity: these facts do not require a foliation of space-time. Does adding a foliation help to any degree at all in determining whether we have an Earth-toMars or a Mars-to-Earth trip? No. For even if we were to add the foliation, the crucial question of which events come first and which later would be unsettled.

So the 'lapse of time' cannot be equivalent to the existence of a foliation.

Maudlin's claim here is that all one needs for a meaningful notion of the passage of time is a distinction between past and future on one's worldline. If that is the case, then one should be able to make sense of tense in all solutions of (e.g.) GR-regardless of whether they do or do not admit of any folation into hypersurfaces of constant time.

Although Maudlin's reasoning here is prima facie tempting, there is an obvious worry: absent a preferred foliation, there can be no observer-independent notion of passage. But presumably, passage is supposed to be an observer-independent matter. Thus, it is not clear that passage in Maudlin's sense can truly be objective passage-it is, rather, purely solipsistic. Indeed, a second worry about Maudlin's strategy is the following: locally in any GR solution, familiar special relativistic effects-most notably the relativity of simultaneity-recur. ${ }^{74}$ But given this, there is no clear notion as to with which events off one's world-line one should be regarded as being simultaneous.

\footnotetext{
72 Mellor (1998).

73 Maudlin (2007, p. 116).

74 For more on the local validity of SR in GR, see e.g. Read et al. (2018).
} 
And given this, Maudlin has not afforded us a clear sense of even the local passage of time.

\section{Concluding remarks}

A-theorists such as Swinburne have sought vindication for their views by turning to GR. In this paper, we have evaluated several objections to this programme. While we have found some to be off-the-mark, others highlight legitimately areas in which further work remains to be done in order to render the A-theorist's approach compelling and watertight. Even this notwithstanding, however, there is no further motivation to be an A-theorist in GR as compared with any other context. Thus, A-theorists cannot appeal to this theory in order to justify their views.

Whither, then, the A-theory? At this juncture, the challenges for that view are more technical than conceptual-in particular, there is an urgent need for precise answers to questions such as the following: (1) how robust against perturbations is the cosmic simultaneity present in FLRW solutions?; (2) how, precisely, can local observers within a galaxy gain access to the fundamental frame of that galaxy? There being compelling affirmative answers to (1) and (2) is, in our view, a necessary condition for anyone to take seriously the general relativistic A-theory. It remains to be seen whether these answers can be provided. ${ }^{75}$

Acknowledgements We are grateful to Max Binkle, Baptiste Le Bihan, Niels Martens, Martin Pickup, Oliver Pooley, Gonzalo Rodriguez-Pereyra, Aviram Rosochotsky, Richard Swinburne, to the anonymous referees, and to the audience of the Oriel College Philosophy Society, for valuable feedback on the contents of this paper.

Open Access This article is licensed under a Creative Commons Attribution 4.0 International License, which permits use, sharing, adaptation, distribution and reproduction in any medium or format, as long as you give appropriate credit to the original author(s) and the source, provide a link to the Creative Commons licence, and indicate if changes were made. The images or other third party material in this article are included in the article's Creative Commons licence, unless indicated otherwise in a credit line to the material. If material is not included in the article's Creative Commons licence and your intended use is not permitted by statutory regulation or exceeds the permitted use, you will need to obtain permission directly from the copyright holder. To view a copy of this licence, visit http://creativecommons.org/licenses/by/4.0/.

\section{References}

Arkani-Hamed, N., Cheng, H.-C., Luty, M., \& Thaler, J. (2005). Universal dynamics of spontaneous Lorentz violation and a new spin-dependent inverse-square law force. Journal of High Energy Physics. https:// doi.org/10.1088/1126-6708/2005/07/029.

Balashov, Y., \& Janssen, M. (2003). Critical notice: Presentism and relativity. British Journal for the Philosophy of Science, 54(2), 237-246.

Belot, G. (2005). Dust, time and symmetry. British Journal for the Philosophy of Science, 56, 255-291.

Bourne, C. (2005). A future for presentism. Oxford: Oxford University Press.

\footnotetext{
75 There does exist some classical literature to which the A-theorist may be able to appeal in order to answer these questions (at least in part)—see e.g. Prokhovnik (1964). Our point, though, is that the A-theorist must explicitly digest and engage with this material, in order to fill the lacunae in her views which we have identified.
} 
Bourne, C. (2010). A future for presentism (pp. 201-202).

Broad, C. D. (1923). Scientific thought. London: Kegan Paul.

Brown, H. R. (2005). Physical relativity: Spacetime structure from a dynamical perspective. Oxford: Oxford University Press.

Brown, H. R., \& Pooley, O. (2006). Minkowski spacetime: A glorious non-entity. In D. Dieks (Ed.), The ontology of spacetime (pp. 67-92). Amsterdam: Elsevier.

Callender, C. (2017). What makes time special?. Oxford: Oxford University Press.

Callender, C., \& McCoy, C. D. (forthcoming). Time in cosmology. In E. Knox \& A. Wilson (Eds.), The routledge companion to the philosophy of physics.

Craig, W. L. (2000a). The tensed theory of time: A critical examination. Dordrecht: Kluwer.

Craig, W. L. (2000b). The tenseless theory of time: A critical examination. Dordrecht: Kluwer Academic Publishers.

Craig, W. L. (2001a). Time and the metaphysics of relativity. Dordrecht: Kluwer.

Craig, W. L. (2001b). God, time, and eternity. Dordrecht: Kluwer.

Curiel, E. (2017). A primer on energy conditions. In D. Lehmkuhl, G. Schiemann, \& E. Scholz (Eds.), Towards a theory of spacetime theories. Birkhäuser: Basel.

Dasgupta, S. (2016). Symmetry as an epistemic notion (twice over). British Journal for the Philosophy of Science, 67, 837-878.

Earman, J. (1995). Bangs, crunches, whimpers and shrieks. Oxford: Oxford University Press.

French, S. (2011). Metaphysical underdetermination: Why worry? Synthese, 180, 205-221.

Gödel, K. (1949). A remark about the relationship between relativity theory and idealistic philosophy. In P. A. Schlipp (Ed.), Albert Einstein: Philosopher-scientist. La Salle, IL: Open Court.

Huggett, N., \& Wüthrich, C. (2013). Emergent spacetime and empirical (in)coherence. Studies in History and Philosophy of Modern Physics, 44, 276-285.

Janis, A. (2018). Conventionality of simultaneity. In E. N. Zalta (Ed.), The Stanford encyclopedia of philosophy.

Jeans, J. (1936). Man and the universe. In J. Jeans, W. Bragg, E. Appleton, E. Mellenby, J. Haldane, \& J. Huxley (Eds.), Scientific progress (pp. 11-38). London: MacMillan.

Knox, E. (2019). Physical relativity from a functionalist perspective. Studies in History and Philosophy of Modern Physics, 67, 118-124.

Le Bihan, B. (2014). No-futurism and metaphysical contingentism. Axiomathes, 24, 483-497.

Malament, D. B. (2012). Topics in the foundations of general relativity and Newtonian gravitation theory. Chicago, IL: University of Chicago Press.

Maudlin, T. (1993). Buckets of water and waves of space: Why spacetime is probably a substance. Philosophy of Science, 60(2), 183-203.

Maudlin, T. (2007). The metaphysics within physics. New York, NY: Oxford University Press.

Maudlin, T. (2012). Philosophy of physics: Space and time. Princeton, NJ: Princeton University Press.

McTaggart, J. M. E. (1908). The unreality of time. Mind, 17, 457-474.

Mellor, D. H. (1998). Real time II. London: Routledge.

Menon, T., Linnemann, N., \& Read, J. (forthcoming). Clocks and chronogeometry: Rotating spacetimes and the relativistic null hypothesis. British Journal for the Philosophy of Science.

Mercati, F. (2018). Shape dynamics: Relativity and relationalism. Oxford: Oxford University Press.

Miller, K. (2009). Defending contingentism in metaphysics. Dialectica, 63(1), 23-49.

Monton, B. (2006). Presentism and quantum gravity. In D. Deiks (Ed.), The ontology of spacetime (pp. 263-280). Boston, MA: Elsevier.

Mozersky, J. (2011). Presentism. In C. Callendar (Ed.), The Oxford handbook to the philosophy of time (pp. 122-144). Oxford: Oxford University Press.

Norton, J. D. (2012). Approximation and idealization: Why the difference matters. Philosophy of Science, 79, 207-232.

Oaklander, L. N. (2012). A-, B-, and R-theories of time: a debate. In A. Bardon (Ed.), The future of the philosophy of time (pp. 1-24). London: Routledge.

Pettini, M. (2018). Introduction to cosmology: Basic concepts. Online lecture notes. https://people.ast.cam. ac.uk/ pettini/Intro\%20Cosmology/.

Pitts, J. B. (2013). Gauge-invariant localization of infinitely many gravitational energies from all possible auxiliary structures. General Relativity and Gravitation, 42, 601-622.

Pooley, O. (2006). Points, particles, and structural realism. In D. Rickles, S. French, \& J. T. Saatsi (Eds.), The structural foundations of quantum gravity (pp. 83-118). Oxford: Oxford University Press. 
Price, H. (1996). Time's arrow and archimedes' point. New York, NY: Oxford University Press.

Prokhovnik, S. J. (1964). A cosmological model of light propagation. Proceedings of the Cambridge Philosophical Society, 60, 265-271.

Read, J. (forthcoming). Functional gravitational energy. British Journal for the Philosophy of Science.

Read, J., Brown, H. R., \& Lehmkuhl, D. (2018). Two miracles of general relativity. Studies in History and Philosophy of Modern Physics, 64, 14-25.

Rosen, G. (2006). The limits of contingency. In F. MacBride (Ed.), Identity and modality (pp. 13-38). Oxford: Oxford University Press.

Skow, B. (2015). Objective becoming. Oxford: Oxford University Press.

Smeenk, C. (2013). Time in cosmology. In A. Bardon \& H. Dyke (Eds.), The Blackwell companion to the philosophy of time (pp. 201-219). Oxford: Blackwell.

Swinburne, R. (1968). Space and time. London: Palgrave MacMillan.

Swinburne, R. (1993). God and time. In E. Stump (Ed.), Reasoned faith (pp. 204-222). London: Cornell University Press.

Swinburne, R. (1996). The beginning of the universe and of time. Canadian Journal of Philosophy, 26(2), $169-189$.

Swinburne, R. (2008). Cosmic simultaneity. In W. L. Craig \& Q. Smith (Eds.), Einstein, relativity and absolute simultaneity (pp. 224-261). London: Routledge.

Wallace, D. (2012). The emergent multiverse. Oxford: Oxford University Press.

Wüthrich, C. (2010). No presentism in quantum gravity. In V. Petkov (Ed.), Space, time, and spacetime, fundamental theories of physics, Vol. 167 (pp. 257-278). Berlin: Springer.

Wüthrich, C. (2013). The fate of presentism in modern physics. In R. Ciunti, K. Miller, \& G. Torrengo (Eds.), New papers on the present-Focus on presentism. Munich: Philosophia Verlag.

Publisher's Note Springer Nature remains neutral with regard to jurisdictional claims in published maps and institutional affiliations. 